Beate Sommerfeld

Adam-Mickiewicz-Universität, Poznań

DOI: $10.19195 / 0435-5865.141 .9$

\title{
„Auf das Material kommt es an“ - Franz Friedrichs Roman Die Meisen von UUsimaa singen nicht mehr als Reflex auf den Umbruch ins digitale Zeitalter
}

Dass Literatur die Entwicklung der Künste reflektierend begleitet und sich vor allem neuen Kunstformen und Medien gegenüber neu positioniert, indem sie sich von ihnen abgrenzt, oder aber mit ihnen interferiert bzw. sich von ihnen affizieren lässt, ist ein Gemeinplatz der Komparatistik (vgl. Degner / Wolf 2010; Zemanek / Nebrig 2012: 166-169). Auch der Umbruch vom analogen zum digitalen Zeitalter wird in der Literatur nachvollzogen und führt zu Umgruppierungen und Wertungsverschiebungen im System der Künste (vgl. Paech / Schröter 2008: 9-12). Ein interessanter Beleg dafür ist der Roman Die Meisen von UUsimaa des Experimentalfilmers und Autors Franz Friedrich (2014).

Der Debütroman Friedrichs wurde von der Kritik äußerst wohlwollend aufgenommen, bereits vorab mit dem Preis der Ponto-Stiftung ausgezeichnet und zudem für die Longlist des Deutschen Buchpreises 2014 nominiert - und dies, obwohl er es seinen Rezipienten nicht gerade leicht macht. Der Text spielt auf drei, kunstvoll ineinander verflochtenen Zeit- und Handlungsebenen, die zunächst unverbunden nebeneinander zu stehen scheinen. Drei Erzählstränge, die jeweils im Abstand von zehn Jahren spielen, ranken sich um die finnische Insel UUsimaa und das Geheimnis ihrer Lapplandmeisen, die eines Tages urplötzlich aufgehört haben zu singen. Da ist zum Auftakt ein Student der Filmwissenschaft, der im Keller seines Instituts einen Dokumentarfilm einer Filmemacherin namens Susanne Sendler betrachtet ein Film, der den gleichen Titel trägt wie der Roman und 1997 während Sendlers Reise auf die Insel entstand, auf der sie das Verstummen der Meisen ergründen möchte. Die einzige Kopie des Films wird durch eine Ungeschicklichkeit während der Vorführung vernichtet. Seine Bilder haben sich dem Studenten aber derart tief ins Gedächtnis gebrannt, dass er sich 2017 nach Uusimaa aufmacht, weil die 
Meisen dort ebenso unvermittelt und rätselhaft ihren Gesang wieder aufgenommen haben. Der zweite Teil des Romans besteht aus dem tagebuchartigen Bericht Susanne Sendlers über ihre Reise auf die Insel. Um es einer Gruppe von Ornithologen zu ermöglichen, das mysteriöse Verstummen der Meisen zu erforschen und zu erklären, wurde die Insel kurz zuvor zwangsevakuiert, und die Filmemacherin trifft auf eine merkwürdige Gesellschaft von Wissenschaftlern und eine in Leere erstarrte Natur. 2007 schließlich wird eine amerikanische Austauschstudentin, die in Fotoarchiven nach Materialien für ihre Dissertation sucht, von einer fremdartigen Sängergemeinde, deren kehlig-vokalischer Gesang von einer verheißenen Insel und ihren Meisen handelt, in den Bann gezogen. Die Erzählstränge sind durch das Rätsel um das Schweigen der Vögel miteinander verklammert. Der Roman eröffnet einen Raum der Spekulationen: wissenschaftliche Arbeiten, in denen mehr oder weniger abstruse Theorien entwickelt werden, finstere endzeitliche Visionen und Prophezeiungen, nüchterne umweltpolitische Analysen, versponnene Mythen und Märchen und eben der Gesang der UUsimaaner spinnen ein dichtes Netz von Texten und Diskursen um das Verstummen der Meisen, die zwischen dem vagen Gefühl, eine Schuld am Verstummen der Natur zu tragen, und der Sehnsucht nach deren Wiedererklingen, die in Mythen und Gesängen aufbewahrt ist, pendeln. Im Zuge der Diskursivierungen wird das Schweigen der Lapplandmeisen zum Sinnbild für die Entfremdung des Menschen von der Natur. Die Projekte, die im Roman in Angriff genommen werden, sind sämtlich in den Rahmen einer Annäherungen an die Natur zu stellen: die Filmprojekte auf der Insel UUsimaa, ein abstruses Aussteigerprojekt in den Wäldern um Stockholm, in dem der Filmstudent mit seiner Freundin den Versuch eines einfachen Lebens unternimmt, ebenso wie die sehnsuchtsvollen Gesänge der Chöre, die eine wiedergewonnene Einheit mit der Natur beschwören.

Auf all ihren Wegen sind die Protagonisten mit Medien gewappnet. Kameras und Fotoapparate, Aufzeichnungsgeräte für Vogelgesang, aber auch Notizblöcke und Tagebücher sind die Requisiten, die die Figuren mit sich führen. Die Dispositive der unterschiedlichen „Aufschreibesysteme“ (Kittler 2003) werden in Friedrichs Roman gegeneinander verrechnet und ausgespielt. Vor allem die thematischen Bezüge zu den Medien Fotografie und Kinematografie durchziehen die Erzählstränge wie ein medientheoretischer Subtext. Im ersten Satz des Romans heißt es über den angehenden Filmemacher: „Er stand hinter dem Projektor im „Kabuff', der Vorführkabine des kleinen Kinos im Keller des Instituts.“ (Friedrich 2014: 9) Film und Kino begleiten den Text bis zum Schluss: Sein letzter Absatz ist eine Nahaufnahme des Gesichts einer der singenden Meisen von UUsimaa. Friedrichs Roman sind aber nicht nur die Spuren einer diskursiven Auseinandersetzung mit den Medien Fotografie und Kinematografie eingeschrieben-der Text zeichnet darüber hinaus präzise eine Phase der Medienentwicklung auf, und zwar den Umbruch vom analogen ins digitale Zeitalter. Als literarischer Reflex auf den medialen Wandel soll der Text im Folgenden gelesen werden. Aus dieser Fokussierung ergeben sich mehrere Fragestellungen: 
Wie verhalten sich die digitalen zu den analogen „Aufschreibesystemen“? Wie positioniert sich Literatur zu den technischen Medien Fotografie und Film? An diesen zwei Achsen, um die sich die medialen Relationen in Friedrichs Roman gruppieren, soll sich die Analyse orientieren.

\section{Analog vs. digital, Berührung vs. Repräsentation}

Der analoge Film erscheint in Friedrichs Text als ein in die mediale Abstellkammer geschobenes Relikt aus einer zum Ableben verurteilten Welt. Für den Status des Abgelegten steht das verstaubte Kabuff im Filminstitut, in dem der Filmstudent die letzte Filmspule auf einem ausrangierten Projektor abspielt, der während der Projektion entzweigeht und dabei noch die Filmkopie zerstört. Eindrücklicher könnte das Ende der analogen Epoche wohl kaum inszeniert werden. Die Protagonisten, die Filmemacherin Susanne Sendler und der Filmstudent, halten jedoch trotz des Medienfortschritts unbeirrt an den Analogmedium fest und stellen sie den digitalen Bildschirmmedien mit ihren Pixeldarstellungen gegenüber:

Die digitalen Kamera, die sich im Kreis seiner Mitstudenten durchgesetzt hatten, produzierten
Bilder, die behaupteten, dem Gefühl des analogen Films nahezukommen, indem sie seine
Weichheit und Farbigkeit imitierten. Ein Trick, eine Lüge, von der sich die Filmstudenten
täuschen ließen. Sie stürzten sich auf die neue Kameratechnologie, verschlangen mit einer
Gleichgültigkeit alles, was vor ihr Objektiv geriet, und orteten die Bilder auf ihren Festplatten,
die virtuelle Müllhalden verpixelter Erinnerungen waren. Erinnerungen in der imitierten Röte
von Technikolor. (Friedrich 2014: 13)

Die digitalen Medien generieren die Auflösung des fixen Bildes, so wie wir es aus Typografie, Fotografie und Film kennen, zugunsten von instabilen, ständig zerfallenden und sich neu zusammensetzenden Lichtimpulsen, die als Bilder keine festen Bedeutungseinheiten darstellen. Das digitale Bild im Sinne einer unverrückbar gegebenen Entität existiert nicht mehr, sondern nur noch als volatiles Datenmaterial (vgl. Hörisch 2001: 76). Der mediale Wandel wird von Friedrich unter dem Blickwinkel der Verlässlichkeit der Medien als Repräsentationssystem, als eine Veränderung der Aufzeichnungssysteme für die Wirklichkeit ins Visier genommen. Der Befund für das digitale Zeitalter ist demnach der einer schleichenden Verunsicherung des Wirklichkeitsbewusstseins, die als Betrug an den Sinnen inszeniert wird. Indem er den medieninduzierten basalen Zweifel an der Wirklichkeit fokussiert, reflektiert der Roman auf den grundlegenden Wandel (bzw. Wechsel) in der Epistemologie und Ontologie des Bildes, der von der digitalen Fotografie verursacht wurde und in seiner ganzen Tragweite erst in Ansätzen erkennbar ist. Die digitalen Bilder erscheinen im Roman als Repräsentanten einer ständig zerfallenden und sich neu zusammensetzenden Spätmoderne, werden zum Schauplatz einer „Ästhetik des Verschwindens“, die von Friedrich Kittler (2002: 40f) namhaft gemacht wurde. Indem gerade ein Dokumentarfilm als Ex- 
empel gewählt wird, wird die Frage nach der Repräsentation von Wirklichkeit ins Zentrum der medienästhetischen Diskussionen gestellt.

Der mediale Umbruch wird im Text aber noch weitergehend funktionalisiert. Es ist Friedrich augenscheinlich darum zu tun, den Wandel im Weltverhältnis aufzuzeigen, den die Digitalmedien mit sich bringen. Wirklichkeit ist nämlich in den digitalen Medien nicht nur ungreifbar, diese bedingen aufgrund ihrer Funktionsweise eine Realitätszertrümmerung: „Um ein digitales Bild zu erzeugen, musste das aufgenommene Objekt zerstückelt und letztlich ausgelöscht werden, ein Massaker an der Welt." (Friedrich 2014: 13) Dieses Zerstören der Wirklichkeit durch ihr Virtualisieren wird schließlich konsequent zur „Sündhaftigkeit“ des digitalen Zeitalters hochstilisiert - so versteigt der Protagonist sich ,immer weiter in eine Theorie, die womöglich haltlos war: Die digitale Welt sei eine Sünde.“ (Friedrich 2014: 35) Friedrichs Roman rekurriert damit auf einen zutiefst skeptischen Diskurs, der eine dominante Spur in der gegenwärtigen Debatte um die neuen Medien bildet. ${ }^{1}$ Seine Zeitdiagnose ist die Entfremdung vom Echten als Signatur der Epoche, die vom Analogen zum Digitalen überging. Sie wird im Schweigen der „Meisen von UUsimaa“ figuriert, in dem sich eine fremde und abweisende, schweigende Welt verdichtet.

Den digitalen Medien mit ihrem Dispositiv des Schwindens der Sinne (Hörisch 2001: 24f) werden die analogen Medien als letztes Residuum schwindender Realitätserfahrung gegenüberstellt. Der Roman bezieht damit stark polarisierend zum Medienwandel Stellung und schlägt sich auf die Seite der Analogmedien Fotografie und Film. Während in den digitalen Medien das Bild nur noch im Modus der Virtualität existiert, ist es im analogen Film und der Analogfotografie materiell präsent und damit den Sinnen verfügbar. Selbst für den analogen Film ist jedes Bild als Entität greifbar, als einzeln herauslösbares Ganzes erfassbar. So ist es möglich, während des Abspielens des Films

den Drehbewegungen im Laufwerk zu folgen und beobachtete, wie sich das Filmband Lage für Lage um die Wickelachse legte. Jedes Bild konnte er sehen, das war das Material. (...) Er könnte den Projektor anhalten und den Film herausnehmen. Er könnte mit seinen Fingern die Perforation am Rand des Bandes entlangfahren und den spezifischen Geruch einatmen. Dies war die Wirklichkeit, der es galt, die Treue zu halten. Auf das Material kam es an. (Friedrich 2014: 12f)

Aufgrund seiner Materialität gelingt es dem analogen Medium, eine verlässliche Spur zur Wirklichkeit zu legen. Indem seine Materialität exponiert wird, reiht sich das Medium Film in die Gegenstände der Welt ein - der Text stellt das Ephemere des Materials aus, das einem natürlichen Verfall unterliegt und gerade darin seine gegenständliche Qualität erweist. Wenn im Roman refrainartig der Satz wiederholt wird: „Auf das Material kommt es an." - so ist damit auch die besondere Genese des fotografischen Bildes gemeint, die einer materiellen Grundlage bedarf - ist doch die

${ }^{1}$ Zum Übergreifen des Virtuellen aufs Ontologische vgl. Ott (2007: 156f); Stiegler (2004: 104-125); Lunenfeld (2002: 158, 65f). 
Fotografie das Ergebnis eines physikalischen Abdrucks, der durch Lichtreflexion auf eine lichtempfindliche Oberfläche übertragen wird. In den analogen Medien tragen die Bilder also die Spur der Ausstrahlung materieller Lichtpartikel an sich, und im Gegenzug lässt sich behaupten, dass am analogen Medium noch die Überreste des Gegenstands haften. Wenn in Friedrichs Text das analoge Bild gegen die digitale Fotografie als ein ,höflich entnommener Abdruck, Licht gebannt durch Staub und Chemie" (Friedrich 2014: 13) ins Feld geführt wird, so nimmt der Roman nicht nur auf physikalisch-technische Sachverhältnisse Bezug, sondern rekurriert auch auf die frühe Phase des Fotografiediskurses, in dem die Fotografie als Abzug der Wirklichkeit wahrgenommen wird. Bereits in den ersten Jahren nach der Erfindung der Fotografie bildet sich ein zumindest implizites Verständnis darüber aus, dass die Besonderheit des fotografischen Bildes darin besteht, eine physische Verbindung zum Dargestellten unterstellen zu dürfen. So schreibt 1839 der Kunstkritiker Eduard Kolloff (1985: 162) über die ersten Daguerrotypien in Paris, sie halte gleichsam „Abdrücke der Natur“ fest: „,ich erstaunte nicht wenig über diese gleichsam vom Himmel gefallenen Abdrücke." Dementsprechend heißt es im ersten fotografisch illustrierten Buch überhaupt The Pencil of Nature (Kemp 1980:62) über die Fo-

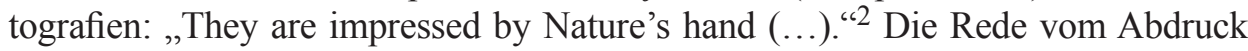
zeichnet eine Linie, die in fotoästhetischen Überlegungen bis heute präsent ist. Den Gedanken, dass die Fotografie als Matrize für Abdrücke Kontiguitätsbeziehungen zwischen Medium und Gegenstand herstellt und zwischen Zeichen und Referenten eine Spur ausfaltet, finden wir in Roland Barthes Essay Die helle Kammer (1985: 90f $)^{3}$ und in neuester Zeit stellt Georges Didi-Huberman den Status der Fotografie als einer Emanation des Gegenstands heraus und kennzeichnet den Abdruck als eine Form der Berührung, der für eine besondere physische Nähe der dargestellten Dinge zum Bildträger steht. Er spricht vom „Berührungspunkt“ als dem Punkt, ,an dem das Bild an das Reale rührt.“ (Didi-Huberman 2007: 113, 207)

In Friedrichs Text wird eine Ästhetik der Berührung entfaltet, die im Charakteristikum der Fotografie ihren Ausgang nimmt, Abdruck des Referenten zu sein. So wird die analoge Fotografie mit ihrer materiellen Angebundenheit, in deren Bil-

2 Die Kunsthistorikerin Rosalind Krauss (1998: 143-145)fasst den frühen Fotografiediskurs wie folgt zusammen: „(...) eine Photographie ist eine photochemisch verfertigte Eindrückung eines Ereignisses - wobei das Ereignis als körperliche Ursache zum photographischen Bild in Beziehung steht. (...) Die Photographie ist, wie gesagt, eine Art Index. Sie produziert die bezeugende Spur der Körper oder der Objekte, die ihren Abdruck in die Emulsion (...) gestempelt haben.“

3 „Die Photographie ist, wörtlich verstanden, eine Emanation des Referenten. Von einem realen Objekt, das einmal da war, sind Strahlen ausgegangen, die mich erreichen, der ich hier bin, (...) die Photographie des verschwundenen Wesens berührt mich wie das Licht eines Sterns. Eine Art Nabelschnur verbindet den Körper des photographierten Gegenstandes mit meinem Blick: das Licht ist hier, obschon ungreifbar, doch ein körperliches Medium, eine Haut, die ich mit diesem oder jener teile, die einmal photographiert worden sind.“

4 Ähnlich schreibt Lethen (2014: 25) vom „Stich“ der Berührung, der vom fotografischen Bild ausgeht. 
dern die Aura der Objekte sich ein letztes Mal verschanzt ${ }^{5}$, in den Passagen inszeniert, in denen die Studentin Monika die Archive der Berliner Bibliothek auf der Suche nach Archivaufnahmen durchforstet, die sie in ihrer Dissertation verwerten möchte. Exponiert wird die materielle Seite der Fotografien, ihre Einmaligkeit und das Hier und Jetzt des Gegenstands, die sie den Sinnen verfügbar macht:

Dick wie Karton war das Papier, das sie zwischen den Fingern hielt, und mit jeder neuen Seite strömte ihr der süßliche Geruch von Buchbindeleim und Druckfarbe entgegen. Drei Farben waren es: Blau, Grün und Rot, die Pigmente erhaben und weich wie Samt, eine Puderschicht, die die Fotografien materialisierte zu einer auf das Papier gebannten Wirklichkeit. Monika konnte sie mit geschlossenen Augen ertasten wie eine Gravur. (Friedrich 2014: 153f)

Es wird in diesem synästhetischen Zugriff auf die Bilder ein berührendes Sehen erprobt, das sich aus der Materialität der Fotografien speist. Die verblichenen Fotos in den archivierten Zeitschriften, an das Medium des Papiers gebunden, ermöglichen durch ihre haptischen Qualitäten ein „Hineingreifen“6 in die Bilder, das auf Empathie angelegt ist und körperliche Resonanzerfahrungen initiiert: „Es war seltsam, Monika glaubte, den Duft zu kennen, den die Frau an dieser Körperstelle ausströmte, sie schmeckte ihn auf der Zunge. (...) Sie kannte diese Frau. (...) und ihre Liebe war groß, sie überwand die schwindelerregende Kluft zwischen Monikas Wirklichkeit und der Gegenwart dieser Frau.“ (Friedrich 2014: 162f) In der Fotografie eines geschundenen Bauarbeiters erblickt sie die leidende Kreatur, mit der sie eine geradezu körperlich schmerzhafte Empathie fühlt. Die Digitalfotografie dagegen vermag dem tastenden Blick keinen Einlass zu gewähren - nachdem Monika mit ihrem Smartphone die Archivaufnahmen abfotografiert hat, wirft sie keinen auch noch so flüchtigen Blick mehr darauf. Auch die Reisen der Filmemacherin Sendler auf die Insel UUsimaa sind ein taktiles Eingreifen in die von ihren materiellen Anbindungen losgelöste Kultur. Das Berühren der Natur wird gegen Virtualität und digitale Flüchtigkeit gestellt:

Ich erinnere mich, ich saß auf einem dicht mit Moos bewachsenen Felsen, der rau und weich zugleich war. Wenn ich das Moos mit der Hand berührte, fühlte es sich an, als streifte ich das Fell eines Tieres, die speckige Wolle eines Schafs. Durch das Pflanzenpolster ließ sich das Gestein mit seinen kleinen Zerklüftungen und Spalten ertasten wie hervorstehende Rippen. Gedankenverloren begann ich den Felsen zu streicheln. (Friedrich 94f)

Auch hier impliziert Berührung das Zustandekommen von Resonanzphänomenen zwischen Subjekt und Welt. Das Angerührt-Werden durch die Natur geht mit einem tastenden Sehen einher, das durch das Medium der Kamera unterstützt wird. So scheinen die durch die Membran der Filmkamera betrachteten Gegenstände der Natur ,in dem Moment des Betrachtens eine Wärme auszustrahlen, die bis hinunter an den Strand reichte, wo ich (die Filmemacherin, B.S.) stand und schon bald die ersten zehn Minuten meines Materials verschossen hatte.“ (Friedrich 2014: 139)

${ }^{5}$ Den Begriff der Aura übernehme ich von Walter Benjamin (1974: 441).

6 „Ein Verlangen überkam sie, sie wollte hineingreifen in das Bild, hinabsteigen in die Vergangenheit und die Menschen auf der Fotografie warnen vor der Zeit." (Friedrich 2014: 158). 
Durch Resonanzeffekte, die im Betrachten das „leibliche Responsorium“ (Waldenfels 1999: 12) aktivieren, entstehen Kontaktzonen zwischen Subjekt und Welt, in denen osmotischer Austausch möglich wird. Die analogen Medien lassen sich von den Gegenständen der Welt berühren und in Schwingungen versetzen. Das Medium Fotografie gilt in Friedrichs Text als Membran, die das Subjekt weniger von seinen Objekten trennt als vielmehr eine Kontaktfläche zwischen ihnen ausbildet. Resonanz wird damit in Friedrichs Text von einem physikalischen Phänomen zu einem Weltverhältnis ausgeweitet. Dabei wird der Schritt vollzogen von der physikalischen Tatsache des Mitschwingens zum sinnhaft erfahrenen Resonanzphänomen, und von dort aus zum ästhetischen Prinzip der Resonanzeffekte als Kontaktflächen des Subjekts mit seiner Umwelt. Resonanz als Mitvibrieren mit den Objekten der Wirklichkeit wird in Friedrichs Roman zum Aufblitzen der Hoffnung auf Anverwandlung und Antwort in einer schweigenden Welt, bezeichnet das Gegenteil von Entfremdung, ein Affiziert-Werden, bei dem ein Wechselprozess in Gang kommt: die Erfahrung des Berührt-, Bewegt- und Ergriffenwerdens. Subjekt und Welt werden in dasselbe Verhältnis zueinander gebracht wie in der Leibnizschen Metapher der zwei Saiten, die auf denselben Ton gestimmt sind (vgl. Bredekamp 2004: 17-23).

Während die digitalen Medien Wirklichkeit zertrümmern und virtualisieren, fixiert das analoge Bild Wirklichkeit und ist dabei Ausdruck des Respekts gegenüber der Welt: „ein höflich entnommener Abdruck.“ (Friedrich 2014: 13) Wie die digitalen induzieren also auch die analogen Medien ein Weltverhältnis: Gegen die „digitale Sünde“ wird das analoge Weltverhältnis ins Feld geführt, das durch Berührung und Abdruck gekennzeichnet ist. Ästhetisches Ideal sind „heilige“ Bilder wie das Schweißtuch der Veronika mit ihren körperlichen Einprägungen:

Die Ikonoklasten der Antike hätten nur dasjenige Bild als heilig anerkannt, auf dem sie einen unmittelbaren Abdruck des Göttlichen sahen. Die Flecken und verschwommenen Farbverläufe auf dem Schweißtuch der Veronika, die sich aus dem Blut Jesu gefärbt haben sollen und einen Abdruck seines Gesichts darstellten, samt den Lücken, die seine Augenhöhlen beschrieben, waren heilig. Eine Darstellung der Maria dagegen, goldverziert auf Edelholz oder Elfenbein, galt als profan. ,Auf das Material kommt es an.' (Friedrich 2014: 34f)

Indem die Berührung des Abdrucks gegen die Repräsentation ausgespielt wird, können die analogen Medien in Friedrichs Roman zur Signatur einer Epoche werden, in der Subjekt und Welt noch nicht voneinander entfremdet waren.

\section{Der Resonanzraum der Stimme vs. Repräsentationssystem Schrift}

Die in Friedrichs Roman entfaltete Ästhetik der Resonanzen und Berührungen, die sich aus dem Unbehagen an der Repräsentation speist, wird emblematisch im Gesang der Meisen von UUsimaa verdichtet. Die Stimme wird als Resonanzphänomen per se ins Spiel gebracht: 
Was hörte ich? Ich hörte die Schwingungen in den Membranen der Syrinx, des Vogelstimmkopfs, der nach einer zur Schilfflöte verhexten Nymphe benannt war. Ich hörte die Reibung der Luft in den Knorpeln und Knochenringen. Ich hörte den Enthusiasmus des Tieres und auch die Angst, die Erschöpfung. Und ich hörte die Atempause zwischen den Lauten, auch das Innehalten war gefahrvoll. (Friedrich 2014: 100).

In der Stimme erklingt die Materialität des Körpers, die der Kehle entsteigt, dem Ort, an dem das Lautmaterial gehärtet und gestanzt wird. Diese Stimme wird in Friedrichs Roman in den Gesängen des Chores der Insulaner laut. Die Zuhörenden lassen sich von diesem Gesang körperlich affektieren und in Schwingungen versetzen:

Das müsste ich filmen, dachte ich, und während ich überlegte, wo ich die Kamera aufstellen würde, fiel mir auf, wie feucht die Zähne im Mund der Sopransängerin waren. In ihrem nassen Glanz erinnerten sie an permuttschimmernde Muscheln. Eine kleine, dunkle Zungenspitze drang heraus und fuhr über die Zahnreihen. Eine Nacktschnecke oder ein Blutegel, der in der roten Kehle der Sängerin haust, dachte ich, und bei diesem Gedanken fasste ich mir an den Hals. Etwas bewegte sich in meiner Luftröhre, ich spürte das Aufhüpfen meines Kehlkopfes, er schien auf die doppelte Größe angeschwollen zu sein. (Friedrich 2014: 142).

Auch die Studentin Monika antwortet auf den Gesang des finnischen Kirchenchores, der von den Lapplandmeisen handelt, mit Resonanzphänomenen, indem sie „mit lautlosen Mundbewegungen versuchte, von den dunkel sirrenden Tiefen zu den hohen sphärischen Tönen aufzusteigen“. (Friedrich 224f) Es ist ein harmonischer Gesang, „der nicht aus Wörtern oder Silben bestand, nur aus einer Reihe von Vokalen, $A-I-O-U^{*}$ (Friedrich 2014: 142):

Monika hörte sie einige Vokale intonieren. Sie sang ein $O$, ein $U$ und dann ein $\ddot{U}$. Mit ihrer Stimme, mit der Zunge und mit den Lippen begann die Sängerin zwischen den Lauten zu gleiten und die Übergänge zu ertasten, und aus ihr, aber zugleich auch nicht aus ihr, erklangen hohe, flötende Töne, Strahlen, die sich über den dunklen Vokalgesang legten wie ein helles, klares Licht, so schön klang der Gesang in Monikas Ohren. (Friedrich 2014: 224)

An diesem Gesang versagen sämtliche „Aufschreibesysteme“ bis hin zu den kompliziertesten Computerprogrammen. (vgl. Friedrich 2014: 101) Auch die Schrift erweist ihr Ungenügen, ist gegenüber dem Gesang defizitär. ${ }^{7}$ In der Utopie der menschlichen Stimme als eines Widerhalls, eines Mittönens klingt das Konzept des Resonanzraums der Stimme an, wie es im Rahmen der Kritik am Logozentrismus der Schrift ins Feld geführt wird (vgl. Derrida 2003: 137f). Im

7 „Aus Vogelbestimmungsbüchern kannte ich die Lautumschreibungen des Lapplandmeisengesangs. (...) Dennoch, die Melodiefolgen der Aufnahmen klangen eher elektrisch als mit Buchstabenlauten verwandt, und ich wunderte mich, wie ungenau und anspruchslos die phonetischen Beschreibungen waren. Ob das Stimmenrepertoire der Meisen treffender mit tit tit oder mit piv piv wiedergegeben wurde, wie ich es in unterschiedlichen Vogelführern oft abweichend gelesen hatte, ich konnte es nicht sagen, und auch die den Lautumschreibungen beiseitegestellten Adjektive krächzend, meckernd oder wuchtelnd erschienen mir vor dem Hintergrund der Aufnahmen als hilflose Versuche der Erfassung einer ästhetischen Welt, vor dessen Fremdartigkeit man kapitulierte und der man sich vielleicht besser mit Bildern und Geschichten annähern sollte.“ (Friedrich 2014: 100f). 
Horizont von Friedrichs Text scheint die Utopie eines „,vokalischen Schreibens“ auf, wie sie von Roland Barthes entfaltet wird, eines Schreibens, das mit der Stimme zusammenfällt und mit ihr identisch wird. Im Gesang der Meisen und Chöre wird eine Sprache laut, bei der man die „Rauheit der Kehle, die Patina der Konsonanten, die Wonne der Vokale, eine ganze Stereophonie der Sinnlichkeit hören kann“ (Barthes 1974: 98). Diese Materialität der Stimme, dessen, „das knirscht, das knistert, das streichelt, das schabt“ (ebd.) wird von Barthes dem Medium des Films zugeschlagen:

Eine bestimmte Kunst der Melodie kann eine Vorstellung von diesem vokalen Schreiben geben; aber da die Melodie tot ist, findet man sie heute vielleicht am ehesten im Film. Der Film braucht nur den Ton der Sprache von ganz nah aufzunehmen (...) und in ihrer ganzen Materialität, in ihrer Sinnlichkeit den Atem, die Rauheit, das Fleisch der Lippen, die ganze Präsenz des menschlichen Mauls hören zu lassen (....), und schon gelingt es ihm, das Signifikat ganz weit weg zu rücken (ebd.).

Dieses Hören in der Losgelöstheit von den Signifikaten inszeniert Friedrichs Roman in den Passagen, in denen die Protagonisten die Worte und Lieder in der fremden, unbekannten finnischen Sprache nicht verstehen oder missverstehen, und ganz auf die sinnliche Wirkung der Sprachlaute zurückgeworfen sind. Mit Bedacht eingesetzt wird von Friedrich die fremdartige Phonetik des Finnischen: Mit ihrem Überangebot an Vokalen, die von „schnalzenden Lauten“ durchsetzt sind, wirken diese Worte wie verdichteter Vogelgesang, wie eine „Geheimsprache“ (Friedrich 2014: 229) aus vulkanischen Tiefen. Die Utopie einer Sprache, die sich an der Stimme orientiert, fällt in Friedrichs Romans zusammen mit der Utopie einer Welt, in der Natur und Menschen die gleiche Sprache sprechen.

\section{Neue Sehwelten eröffnen - die Medien und das Optisch-Unbewusste}

Wie ist die nun die Rolle der Medien bei den Annäherungen an die Natur zu veranschlagen? Wenn die Filmemacher mit ihren vorsintflutlich anmutenden, sperrigen Gerätschaften die finnische Natur bereisen, erzeugt der durch die Kamera mediatisierte Blick eine größere Nähe zur Natur als das unbewaffnete Auge sie zu gewähren vermag. Dabei wird das mediale Dispositiv der Genauigkeit bei der Wirklichkeitswiedergabe ins Spiel gebracht.

Mit dem Teleobjektiv unternahm ich einen neuen Versuch. Ich schwenkte die Böschung hinauf zu dem Saum des Weidenwaldes und filmte das silbrige Flimmern der Blätter in den Baumkronen. Mein Blick sank tiefer und schob sich so dicht an Grashalmen, Rainfarnstengeln und bemoosten Wurzelvorsprüngen vorbei, dass ich mir vorzustellen begann, mein Schauen würde das Geräusch brechender Zweige erzeugen. Ich filmte am Gestrüpp des Waldbodens entlang, das sich von einer anlandigen Böe gekrümmt vom Meer und von mir abwandte, bis ich die Kamera schließlich vor einem dickbäuchigen Pilz zur Ruhe kommen ließ, der mich mit seinem 
tintenblauen Hut und seinen weißen, an Papierseiten erinnernden Lamellen in den Bann zog. (Friedrich 2014: 139)

Das Teleobjektiv mit der Möglichkeit von Nahaufnahmen kompensiert das defizitäre Wahrnehmungsvermögen, holen Details in Bild, die der menschlichen Wahrnehmung erst durch die apparative Wahrnehmung erkenntlich werden. Der Natur wird so eigentlich erst durch die medialen Zurüstungen zum Erscheinen verholfen. Dabei nimmt die Beziehung zwischen Auge und optischem Gerät eine metonymische Form an - beide greifen auf der gleichen Funktionsebene ineinander. Die Natur gibt den Rhythmus der Sequenzen vor, der das Auge nicht überfordert. Es wird ein zirkulärer und sukzessiver Aneignungsprozess in Gang gebracht, in dem die Kamera dem menschlichen Auge vorauseilt, jeder Zugewinn neuer Bilder aber mit neuen Unschärfezonen erkauft wird. In diesem Prozess wird das Repräsentationsparadigma überwunden und Wirklichkeit nicht dokumentiert, sondern neu erschaffen: „Susanne Sendler behandelte die Realität schöpferisch. Sie lief ihr nicht hinterher, sie erschuf sie neu." (Friedrich 2014: 12)

Wenn Friedrichs Roman das Potenzial der technischen Medien herausstellt, etwas sichtbar zu machen, was anders nicht beobachtbar ist (eine verborgene oder unbewusste Realität), greift er auch darin auf den frühen, emphatischen Mediendiskurs zurück. Die Leistungen des Mediums Fotografie sind von Anfang an mit einer Sichtbarmachung verbunden, die über eine reine Wiederholung oder Verdoppelung dessen, was das menschliche Auge sehen kann, hinausgeht (vgl. Renner 2005: 105f). Es handelt sich um ein Sichtbarwerden durch das Medium, das immer neue Dimensionen der Wirklichkeit eröffnet: „,wir entdecken immer neue, immer köstlichere Einzelheiten und unendlich viele Feinheiten (...), welche dem unbewaffneten Auge in der Wirklichkeit entschlüpfen." schreibt Eduard Kolloff (1985: 162) über den Detailrealismus der ersten Fotografien. Diese detailgenaue Aufzeichnung des Sichtbaren in einem schöpferischen Sehen hat Walter Benjamin das „Optisch-Unbewusste“ genannt und in der Kleinen Geschichte der Photographie und seinem Kunstwerk-Aufsatz entwickelt. Fotografie und Film - so Benjamins Hoffnung - öffnen die Wahrnehmung auf neue Felder, schaffen neue Räume visueller Wahrnehmung, machen das Optisch-Unbewusste sichtbar, das vorher nicht zugänglich war: „Es ist ja eine andere Natur, welche zur Kamera als zum Auge spricht; (...) Die Photographie mit ihren Hilfsmitteln: Zeitlupen, Vergrößerungen erschließt sie ihm. Von diesem Optisch-Unbewußten erfährt er erst durch sie (...).“(Benjamin 1980: 371; 1974: 500) Der Film bietet dank seiner formalästhetischen Mittel eine neue Sicht auf die Dinge, indem er das Gefilmte unterbrechen und isolieren, dehnen und raffen, vergrößern und verkleinern kann. Die technischen Medien konservieren Bilder und Töne also nicht nur, sondern verleihen ihnen erst Präsenz und Prägnanz. Dass die medialen Bilder eindrücklicher sind als die ungefilterte Realität, spiegelt sich in Friedrichs Roman nicht zuletzt in den subtilen Übergängen der Erzählweise wider: Während die Bilder 
der Realität eigentümlich vage und schwach konturiert sind, zeichnen sich die Beschreibungen der Fotografien und Filme durch hohe Prägnanz aus. Gleich zu Beginn der Romanhandlung wird das Potenzial des Films erprobt, sein Vermögen, die Welt anders zu sehen als es mit unbewaffnetem Auge möglich wäre. Die wahrnehmungssteigernde Potenzialität erschöpft sich jedoch nicht in einer Vertiefung der Apperzeption, der Überschuss an Wahrnehmung, der durch filmische Mittel wie Bildschnitte, langsame Kamerafahrten, Zeitlupe und Nahaufnahmen erzeugt wird, leitet ins Imaginäre hinüber: „Der Film begann mit einer Fahrt entlang einer Wandvertäfelung aus grünlasiertem Holz. Anstelle einer Eröffnung mit Luftaufnahmen von Küstenlinien und kalbenden Gletschern, wie sie in Naturdokumentationen üblich war, folgte die Kamera der Maserung der Bretter. Aus Rissen, Ringen und Adern entstand eine Seenlandschaft.“ (Friedrich 2014: 10) In den Exkursionen seiner Figuren setzt Friedrich die Arbeit des Filmens in Szene, die dank ,ihrer intensivsten Durchdringung mit der Apparatur (...) tief ins Gewebe der Gegebenheit" einzudringen vermag (Benjamin 1974: 496) und damit neue Dimensionen der Wirklichkeit erschließt, „Bildwelten, welche im Kleinsten wohnen, deutbar und verborgen genug, um in Wachträumen Unterschlupf gefunden zu haben“ (Benjamin 1980: 371).

Die Kamera seziert also nicht nur unsichtbare, kleinste Teile und Schichten, die dem menschlichen Wahrnehmungsvermögen verborgen bleiben, vielmehr sind ihre Art aufzuzeichnen und deren Produkte dem Träumen ähnlich. Wie der Traum macht die Kameratechnik Dinge und Zusammenhänge sichtbar, ein Dazwischen, das sonst im Unsichtbaren residiert. Das Herauslösen des Einzelbildes aus dem Kontinuum als das „Zwischen“ entspricht der Wahrnehmung der Halluzination und des Traums. In den Schauerlebnissen der Romanfiguren ist die Seinsweise des Referenten oftmals eine rein phantasmagorische. ${ }^{8}$ Solcherlei Wachträume überkommen Monika beim Betrachten der Achivfotos: „Mit der Fingerkuppe berührte sie die Fotografie, jede Linie wollte sie sich merken, sich einprägen wie einen Zahlencode, bis Monika sie schließlich vor sich sah, ein Nachbild hinter den geschlossenen Lidern." (Friedrich 2014: 163) Der Text entfaltet eine halluzinatorische Bildlichkeit, die einander überlagernden medialen Repräsentationen verschwimmen zu einer traumhaften Präsenz, die sich über die Realitätswahrnehmung legt und sie mit neuen Gehalten anreichert, letztlich aber ausblendet. Zunehmend schieben sich die medialen Bilder vor die Wirklichkeit - die Studentin Monika findet schließlich nicht mehr den Weg aus einer phantastischen Wirklichkeit und verliert sich in der Intensität der Archivfotografien bis zum Wahnsinn, der die Gegenstände der Realität zu Phantomen der Bilder werden lässt, und dem Filmstudenten, der sich zu Beginn der Romanhandlung den Dokumentarfilm von Susanne Sendler anschaut, brennen sich die medialen Bilder so tief ins Gedächtnis, dass

${ }^{8}$ Zur phantasmagorischen Präsenz des Referenten in der Fotografie vgl. von Amelunxen (1995: 213f). 
er sich Jahre später - Frau und Kind zurücklassend - auf den Weg nach UUsimaa macht. Die Fahrt mit der zur Insel übersetzenden Fähre wird zu einer Reise in eine traumumwobene Wirklichkeit. Auf einer weißen Leinwand erscheint die den Text abschließende Nahaufnahme einer singenden Meise, von der wir nicht wissen, ob sie der Erzähler tatsächlich sieht oder ob sie seiner Einbildung entsprungen ist. Die Dynamik des Unbewussten greift auf die gesamte Szenerie über, die sich als phantasmatische gleichsam ins Filmband einfaltet. Friedrich erkennt die traumanaloge Dynamik der bewegten Bilder und führt vor, wie die medial gesteigerte Wahrnehmung imaginäre Räume zu eröffnen imstande ist. Das Romanende öffnet den Text auf das Optisch-Unbewusste, jene der gewöhnlichen und medial unbewehrten Wahrnehmung nicht zugänglichen Zwischenwelten. Als Trugbild hat das Gesicht des Vogels den Status des Oszillierens, Changierens zwischen dem Aktuellen und Virtuellen, seine simulakrenhafte Konstitution lässt die Herkunft aus dem Unbewussten erahnen. Zugleich wird das Trugbild erkennbar als ein in sich paradoxer - weil bildloser - Bildgenerator, womit das Denken der Repräsentation entkräftet wird. In der abschließenden Großaufnahme, der das Pathos von Balázs Filmtheorie anhaftet, wird noch einmal eindrucksvoll einer Ästhetik der Berührung das Wort geredet, die sich dem „Analogiezauber“ (Cassirer 1987: 87) des Films verdankt. In einem betörenden Schlussakkord setzt Friedrichs Roman der Ungreifbarkeit digital beschleunigter Bilderfluten noch einmal das Arretieren von Einzelbildern entgegen und sondiert damit die wahrnehmungssteigernden Potenziale technisch vermittelten Sehens. Das Isolieren von Einzelbildern mit technischen Mitteln soll eine „mystische Partizipation“ ermöglichen, wie sie vom Filmtheoretiker Lucien Lévy-Bruhl für das Filmmedium in Anschlag gebracht wird. ${ }^{9}$ In einem herausgehobenen Bewusstseinszustand, der dem des Traums ähnlich ist, wird der Blick des Betrachters in das verstörende Tableau hineingesogen - in einer sprachlosen, nur noch von Gesang erfüllten Nähezone.

\section{Fazit}

Friedrichs Roman skizziert den medieninduzierten Wandel im Weltverhältnis und will die Entwicklung der Sinne auf die Abfolge von einander ablösenden Leitmedien zurückbeziehen. Der Text zeigt die Veränderlichkeit der poetischen, visuellen, akustischen Einbildungskraft durch die Medienentwicklung und spiegelt sie im Medium der Literatur. Dem Roman liegt das Konzept einer subjektiven und zugleich medial inszenierten Imagination zugrunde. Es werden Szenarien der Sinnlichkeit und zugleich intermedialer Reflexion entworfen, sinnliche und mediale Wahrnehmung kunstvoll ineinander verschachtelt. Friedrichs Roman erkundet auf diese Weise das mediale Dazwischen der visuellen Medien Fotografie und Kinematogra-

${ }^{9}$ Zur Filmtheorie von Lévy-Bruhl und seiner Rezeption der in der ersten Hälfte des 20. Jahrhunderts vgl. Sommerfeld (2013: 154-167; 2011: 288f). 
fie und der Literatur. Die technischen Medien werden zum Katalysator poetologischer Reflexion - im Rekurs auf sie soll der Blick für das eigene Medium, die Literatur, geschärft werden.

Friedrichs Roman scheint einen Beleg für die Tendenz zu liefern, die analogen Medien in einer Zeit, in der sie ins Museum der Geschichte abgeschoben werden, im Medium der Literatur wiederkehren und selbstreferent werden zu lassen (vgl. Weixler 2008: 35). Durch die Medienkonkurrenz werden sie sich ihrer spezifischen Ausdrucksmittel bewusst. Im Verhandeln der Mediendispositive greift Friedrich auf den Fotografie- und Filmdiskurs des 20. Jahrhunderts (von Béla Balázs, Lucien Lévy-Bruhl, Walter Benjamin bis hin zu Roland Barthes und Georges Didi-Huberman) zurück. Indem der Roman ein Gewebe aus photoästhetischen Topoi, Metaphern und Diskursen spinnt, modelliert er eine Ästhetik des Abdrucks und der Berührung, in der das Medium als Membran und Kontaktfläche gefasst wird, die die Subjekt-Objektgrenzen zum Vibrieren bringt. Es mag sich darin zum einen eine melancholisch gefärbte Sehnsucht nach dem Gegenstand in seiner Sinnfälligkeit aussprechen, ein Bestreben, Spuren des Lebens im Leblos-Materiellen ausfindig zu machen, und zum anderen ein Unbehagen an der Repräsentation äußern - wird die Spur doch von Friedrich als körperlicher Abdruck, als Einprägung einer Berührung ins Feld geführt. Wie Didi-Huberman geht Friedrich dem Wunsch nach materieller Berührung bis zu dem Punkt nach, wo er sich nicht mehr im Abdruck materialisiert, sondern nur noch im Medium der Schrift ins Reich der Vorstellungen geleitet werden kann. Friedrichs Roman steht im Zeichen einer Selbsterkundung und Selbstbespiegelung des Textes als Bühne der Repräsentation. Indem der Text eine Poetik der Resonanzen entfaltet, soll die Magie des Analogen in die Literatur übertragen werden. In seinem bild- und klangintensiven Erzählen lässt sich Friedrichs Roman von den Fotografie und Film affizieren und macht sich zum Schauplatz von Resonanzphänomenen, wird zum Resonanzkörper, in dem die Stimmen der Welt widerhallen, verstärkt werden und an Prägnanz und Präsenz gewinnen sollen.

\section{Literatur}

Amelunxen, Hubertus von: Photographie und Literatur. In: Zima, Peter V. (Hrsg.): Literatur intermedial. Musik - Malerie - Photograpie - Film. Darmstadt 1995, S. 209-231.

Barthes, Roland: Die Lust am Text. Aus dem Französischen von Traugott König. Frankfurt am Main 1974.

Barthes, Roland: Die helle Kammer. Bemerkung zur Photographie. Aus dem Französischen von Dietrich Leube. Frankfurt am Main 1985.

Benjamin, Walter: Das Kunstwerk im Zeitalter seiner technischen Reproduzierbarkeit. In: Ders.: Gesammelte Schriften. Hrsg. von Rolf Tiedemann und Hermann Schweppenhäuser. Bd. I.2. Frankfurt am Main 1974, S. 435-508.

Benjamin, Walter: Kleine Geschichte der Photographie. In: Ders.: Gesammelte Schriften. Hrsg. von Rolf Tiedemann und Hermann Schweppenhäuser. Bd. II.1. Frankfurt am Main 1980, S. 368-385. 
Bredekamp, Horst: Die Fenster der Monade: Gottfried Wilhelm Leibniz'Theater der Natur und Kunst. Berlin 2004.

Cassirer, Ernst: Das mythische Denken. Philosophie der symbolischen Formen. 2. Darmstadt 1987.

Degner, Uta / Wolf, Christian (Hrsg.): Der neue Wettstreit der Künste. Legitimation und Dominanz im Zeichen der Intermedialität. Bielefeld 2010.

Derrida, Jacques: Die Stimme und das Phänomen. Einführung in das Problem des Zeichens in der Phänomenologie Husserls. Aus dem Französischen von Hans-Dieter Gondeck. Frankfurt am Main 2003.

Didi-Huberman, Georges: Bilder trotz allem. Aus dem Französischen von Peter Greimer. München 2007.

Friedrich, Franz: Die Meisen von UUsimaa singen nicht mehr. Frankfurt am Main 2014.

Hörisch, Jochen: Der Sinn und die Sinne. Eine Geschichte der Medien. Frankfurt am Main 2001.

Kittler, Friedrich: Optische Medien. Berliner Vorlesung 1999. Berlin 2002.

Kittler, Friedrich: Aufschreibesysteme 1800-1900 (1985). München 2003.

Kemp, Wolfgang: Theorie der Photographie I. 1839-1912. München 1980.

Kolloff, Eduard: Der Detailrealismus der ersten Photographien. In: Plumpe, Gerhard (Hrsg.): Theorie des bürgerlichen Realismus. Eine Textsammlung. Stuttgart 1985, S. 161-164.

Krauss, Rosalind: Das Photographische. Eine Theorie der Abstände. München 1998.

Lethen, Helmuth: Der Schatten der Fotografie. Bilder und ihre Wirklichkeit. Berlin 2014.

Lunenfeld, Peter: Digitale Fotografie. Das dubitative Bild. In: Wolf, Herta (Hrsg.): Paradigma Fotografie. Fotokritik am Ende des fotografischen Zeitalters. Bd. 1. Frankfurt am Main 2002, S.S. $158-177$.

Ott, Michaela: Von ontologischen zum digitalen Virtuellen. In: Leitner, Birgit / Engell, Lorenz (Hrsg.): Philosophie des Films. Weimar 2007, S. 156-179.

Paech, Joachim / Schröter, Jens: Intermedialität analog/ digital. Ein Vorwort. In: Dies. (Hrsg.): Intermedialität analog/digital. Theorien-Methoden-Analysen. München 2008, S. 9-12.

Renner, Ursula: „Details sollten sein wie jener Blitz bei Dickens “- Photopoetische Reflexe um 1900. In: Pfotenhauer Helmut/ Riedel, Wolfgang/ Schneider, Sabine (Hrsg.): Poetik der Evidenz. Die Herausforderung der Bilder in der Literatur um 1900. Würzburg 2005, S. 103-127.

Sommerfeld, Beate: Zwischen Augenblicksnotat und Lebensbilanz. Die Tagebuchaufzeichungen Hugo von Hofmannsthals, Robert Musils und Franz Kafkas. Frankfurt am Main u.a. 2013.

Sommerfeld, Beate: Das , Triëdern “ als Textstrategie in Robert Musils Tagebüchern. In: Convivium. Germanistisches Jahrbuch Polen (2011), S. 271-300.

Stiegler, Bernd: Digitale Photographie als epistemologischer Bruch und historische Wende. In: Engell, Lorenz / Neitzel, Britta (Hrsg.): Das Gesicht der Welt. Medien in der digitalen Kultur. München 2004, S. 104-125.

Waldenfels, Bernhard: Sinnesschwellen. Studien zur Phänomenologie des Fremden 3. Frankfurt am Main 1999.

Weixler, Antonius: „Die optische Kluft“. Vor-Augen-Stellen in Carl Einsteins Poetik des Transvisuellen. In: Focus on German Studies 15 (2008), S. 35-59.

Zemanek, Evi / Nebrig, Alexander: Komparatistik. Berlin 2012.

\section{Abstracts}

Der Artikel behandelt den Roman Die Meisen von UUsimaa singen nicht mehr (2014) des Experimentalfilmers und Autors Franz Friedrich als Reflex auf den Umbruch vom analogen zum digitalen Zeitalter. Stark polarisierend bezieht der Roman zum Medienwandel Stellung und schlägt sich auf die Seite der Analogmedien Fotografie und Film, wobei er auf den Fotografie- und Filmdiskurs des 
20. Jahrhunderts (von Bela Balazs, Walter Benjamin bis hin zu Roland Barthes und Georges Didi-Huberman) zurückgreift. Indem der Roman ein Gewebe aus photoästhetischen Topoi, Metaphern und Diskursen spinnt, modelliert er eine Ästhetik des Abdrucks und der Berührung, die auf Roland Barthes' Modell des "vokalischen Schreibens" rekurriert. Das Unbehagen an der Repräsentation, das aus Friedrichs Roman spricht, geht mit einer nostalgisch gefärbten Sehnsucht nach dem Authentischen einher.

Schlüsselwörter: Franz Friedrich, digitales Zeitalter, Medienreflektion, Intermedialität, Fotografieund Filmdiskurs

\section{"The material matters" - reflections on the upheaval from analogue to digital media in the novel The Tits of Uusimaa Don't Sing Any More by Franz Friedrich}

The purpose of the article is to show how literary texts reflect upon the upheaval from analogue to digital media using the example of the novel The Tits of Uusimaa Don't Sing Any More by the experimental filmmaker and author Franz Friedrich (2014). Friedrich approaches the technological shift from analogue to digital and the transforming landscape of media from a critical viewpoint by looking back at the early 20th-century scenario of intermedial exchange. Doing so, he refers to the 20th-century media discourse (Béla Balázs, Walter Benjamin to Roland Barthes and Georges Didi-Huberman), scrutinizing and redefining analogue media by referring to various topoi, metaphors (the analogue as a mental imprint of the real). Friedrich confronts the representation paradigm of literature to the aesthetic of contact and resonances, strongly related to Roland Barthes' concept of "vocal writing".

Keywords: Franz Friedrich, digital revolution, media, intermediality, discourse of photography and film

Beate Sommerfeld

Uniwersytet Adama Mickiewicza

Instytut Filologii Germańskiej

Al. Niepodległości 4

61-874 Poznań

Polen

E-Mail: bsommer@amu.edu.pl 